\title{
Icariin improves acute kidney injury and proteinuria in a rat model of pregnancy-induced hypertension
}

\author{
WENYU ZHANG, WEI YUAN, NING XU, JINPING LI and WENXIU CHANG \\ Department of Nephrology, Tianjin First Central Hospital, Tianjin 300192, P.R. China
}

Received June 26, 2016; Accepted April 25, 2017

DOI: $10.3892 / \mathrm{mmr} .2017 .7513$

\begin{abstract}
Icariin has previously been demonstrated to attenuate hyperglycemia-induced renal injury, however the renoprotective effects of icariin in a rat model of pregnancy-induced hypertension (PIH) remain to be elucidated. The present study aimed to investigate the effect of icariin on PIH-induced acute kidney injury (AKI) and proteinuria. Following 18 days of icariin treatment between day 1 and day 18 of gestation, which was combined with NG-nitro-L-arginine methyl ester (L-NAME) treatment between day 12 and day 18 of gestation to induce $\mathrm{PIH}$, the $24 \mathrm{~h}$ urine protein level, blood urea nitrogen and serum creatinine were measured by using the Coomassie Brilliant Blue method, a commercial enzymatic kit and the picric acid method, respectively. Renal tissues were collected at day 18 of gestation for hematoxylin and eosin staining and immunohistochemistry. The mRNA expression of AGT and protein expression of angiotensin II (Ang II) in the kidneys of control and $\mathrm{PIH}$ rats was investigated by reverse transcription-quantitative polymerase chain reaction and western blot analysis, respectively, to determine the effect of icariin on components of the renin-angiotensin system. The results demonstrated that L-NAME treatment in pregnant rats resulted in significant increases in systolic blood pressure (SBP) and diastolic blood pressure, in addition to the induction of severe proteinuria. The significant increase in SBP and proteinuria in PIH rats was prevented by icariin. L-NAME-induced AKI resulted in profound renal histological alterations, including mesangial expansion and glomerular lesions. L-NAME administration exerted a marked decrease in the mRNA and protein expression levels of nephrin in the kidneys from PIH rats compared with control group. Furthermore, upregulation of circulating and renal Ang II levels in PIH rats was observed. However, icariin treatment significantly reversed the L-NAME-induced downregulation of nephrin and upregulation of circulating and
\end{abstract}

Correspondence to: Dr Wenxiu Chang, Department of Nephrology, Tianjin First Central Hospital, 24 Fukang Road, Tianjin 300192, P.R. China

E-mail: tjzwy1112@163.com

Key words: icariin, pregnancy, hypertension, proteinuria, renal injury renal Ang II levels in PIH rats. These results demonstrated that icariin administration improved urinary protein excretion levels and renal tissue damage in PIH rats, and the underlying mechanism was mediated in part, via upregulation of nephrin expression and downregulation of Ang II.

\section{Introduction}

Hypertensive disorders occur in $\sim 7 \%$ of all pregnancies and are the primary factors that lead to maternal-fetal mortality (1). There is clinical and experimental evidence suggesting that gestational hypertension is associated with the induction of acute kidney injury (AKI) $(2,3)$. A recent literary review indicates that hypertension during pregnancy $(49.2 \%)$ and postpartum hemorrhage $(13.8 \%)$ are the primary factors resulting in pregnancy-associated AKI in China (4). Furthermore, gestational hypertension is associated with proteinuria during pregnancy, which results in fetal growth restriction and low birth weight $(2,5)$. Increased proteinuria may become nephrotic at an early stage during the pregnancy, with an increased risk of intravascular volume depletion, thromboses, reflux nephropathy and patients are additionally at a greater risk of developing urinary tract infections during this period (6). Therefore, it is important to develop safe and effective therapeutic drugs to improve AKI and proteinuria during pregnancy-induced hypertension.

Icariin, the primary active flavonol glucoside in Epimedium, has been widely used therapeutically, due to its anti-tumor effects (7). It has additionally been used in anti-osteoporotic therapy $(8,9)$ and has been demonstrated to delay cellular senescence (10). Icariin ameliorates chemotherapeutic drug induced AKI (11) and protects against 5/6 nephrectomy-induced chronic kidney failure by increasing the number of renal stem cells in a rat model (12). Furthermore, icariin alleviates high glucose-induced type IV collagen and fibronectin accumulation in glomerular mesangial cells (13). These data suggest that the renoprotective properties of icariin have been verified by pharmacological experiments in vivo and in vitro. However, the mechanism of the renoprotection of icariin in a rat model of pregnancy-induced hypertension has not been fully elucidated. To the best of our knowledge, this study is the first to attempt to determine the protective effect of icariin in a rat model of pregnancy-induced hypertension. The data provide evidence that icariin improves AKI and proteinuria during pregnancy accompanied with hypertension. 


\section{Materials and methods}

Animal treatment. Specific pathogen-free experimental animals were obtained from Vital River Laboratories Co., Ltd (Beijing, China). The rats were caged individually under controlled temperature $\left(23 \pm 2^{\circ} \mathrm{C}\right)$ and humidity $(55 \pm 5 \%)$ with an artificial 12-h light/dark cycle, and were given free access to food and tap water. Wistar rats (40 female, 20 male; age 10-12 weeks; weight 180-220 g) were used for the present study. All experimental procedures were carried out in accordance with the Guide for the Care and Use of Laboratory Animals of the National Institutes of Health (14). All protocols were approved by the Animal Care and Research Committee of Tianjin First Central Hospital (Tianjin, China; Permit number: E20130825-003A).

Estrous female rats were mated overnight with one male. The next morning, the presence of a vaginal plug indicated successful mating and was documented as day 0 of gestation. A total of 40 female pregnant rats were randomly divided into five groups: Control pregnant rats, pregnancy-induced hypertension (PIH) rats, PIH rats + icariin $[10 \mathrm{mg} / \mathrm{kg}$, low (L)], PIH rats + icariin $[50 \mathrm{mg} / \mathrm{kg}$, medium (M)] and PIH rats + icariin $[100 \mathrm{mg} / \mathrm{kg}$, high $(\mathrm{H})]$. Nitric oxide synthase inhibitor NG-nitro-L-arginine methylester (L-NAME; Cayman Chemical Company, Ann Arbor, MI, USA); 0.5 g/l drinking water) was administered from day 12 of gestation to induce PIH. Icariin was administered intragastrically from day 1 to day 18 of gestation.

Blood pressure measurement. Systolic blood pressure (SBP) and diastolic blood pressure (DBP) in pregnant rats were measured at day 1 and day 18 of gestation with the $\mathrm{CODA}^{\mathrm{TM} 2}$ non-invasive single channel blood pressure measuring instrument (Shanghai Zande Medical Devices Co., Ltd., Shanghai, China).

Urinary protein detection in serum and plasma. Rats were selected randomly from each group and were placed in metabolic cages to collect urine for $24 \mathrm{~h}$. The total volume of urine was recorded and used to detect urinary total protein and concentration. Urine protein concentration was measured using the Coomassie Brilliant Blue method, serum creatinine was measured using the picric acid method and blood urea nitrogen (BUN) was measured via an enzymatic kinetic method using commercial kits purchased from Nanjing Jiancheng Biological Engineering Research Institute (Nanjing, China). Plasma levels of angiotensin II (Ang II) were detected using a bioactive Ang II ELISA assay (catalog no. C506065; Sangon Biotech, Co., Ltd., Shanghai, China), according to the manufacturer's protocol.

Hematoxylin \& eosin $(H \& E)$ and immunohistochemical staining. Kidney tissues were collected at day 18 of gestation by intraperitoneal injection of sodium pentobarbital (2\%; 200 mg/kg; Sigma-Aldrich; Merck KGaA, Darmstadt, Germany) and were fixed with $4 \%$ formalin at room temperature for $24 \mathrm{~h}$ and paraffin-embedded. Tissues were then cut into $\sim 5 \mu \mathrm{m}$-thick sections, which were stained with $\mathrm{H} \& \mathrm{E}$ at room temperature for 1-2 min and visualized under a microscope (Leica DM 2500; Leica Microsystems GmbH, Wetzlar, Germany).
The immunohistochemical staining analysis of kidney tissue was evaluated using anti-rat Ang II (catalog no. sc-20718; 1:50; Santa Cruz Biotechnology, Inc., Dallas, TX, USA). In brief, the paraffin sections $(5 \mu \mathrm{m})$ were heated in an oven at $65^{\circ} \mathrm{C}$ for $24 \mathrm{~h}$, dewaxed with water and rinsed with PBS for 5 min, 3 times. Paraffin sections were then placed in EDTA buffer (Beyotime Institute of Biotechnology, Haimen, China) for microwave antigen retrieval and boiled for $10 \mathrm{~min}$. Following natural cooling, the sections were washed with PBS 3 times. The sections were placed into $3 \%$ hydrogen peroxide solution and incubated at room temperature for $10 \mathrm{~min}$, to block endogenous peroxidase activity, and then washed with PBS 3 times. They were then blocked with 5\% bovine serum albumin (BSA; Beyotime Institute of Biotechnology) for $20 \mathrm{~min}$ at room temperature, following drying. Following removal of BSA liquid, each section was incubated with $50 \mu 1$ diluted anti-rat Ang II primary antibody overnight at $4^{\circ} \mathrm{C}$, then washed with PBS 3 times. Following the removal of PBS liquid, each slice was incubated with 50-100 $\mu$ l goat anti-rabbit horseradish peroxidase-conjugated secondary antibody (catalog no. sc-2004; 1:2,000; Santa Cruz Biotechnology, Inc.) at $4^{\circ} \mathrm{C}$ for $50 \mathrm{~min}$, then washed with PBS 3 times. A total of 50-100 $\mu \mathrm{l}$ freshly prepared DAB solution was added to each sample. Following washing, sections were counterstained with hematoxylin at room temperature for $5 \mathrm{~min}$, rinsed with tap water, dehydrated, mounted and visualized under a microscope (Leica DM 2500; Leica Microsystems GmbH).

Reverse transcription-quantitative polymerase chain reaction $(R T-q P C R)$. The renal total RNA extraction was performed using TRIzol ${ }^{\circledR}$, according to the manufacturer's protocol (Invitrogen; Thermo Fisher Scientific, Inc., Waltham, MA, USA). Synthesis of cDNAs was performed by RT reactions with $2 \mu \mathrm{g}$ total RNA using moloney murine leukemia virus reverse transcriptase (Invitrogen; Thermo Fisher Scientific, Inc.) with oligo dT 15 primers (Fermentas; Thermo Fisher Scientific, Inc.) and $4 \mu 1$ Maxima 5X Reaction Mix (Invitrogen; Thermo Fisher Scientific, Inc.), according to the manufacturer's protocol. The RT temperature protocol was as follows: $37^{\circ} \mathrm{C}$ for $50 \mathrm{~min}$ and $70^{\circ} \mathrm{C}$ for $15 \mathrm{~min}$. The first strand cDNAs served as the template for the PCR, performed using a DNA Engine (ABI 7300; Thermo Fisher Scientific, Inc.). GAPDH served as an internal control, and was used to normalize the data to determine the relative expression of the target genes using the $2^{-\triangle \Delta C q}$ method (15). Reaction mixtures $(20 \mu \mathrm{l})$ were prepared using the TaqMan Universal PCR Master Mix (Thermo Fisher Scientific, Inc.). The reaction conditions were set according to the manufacturer's protocol. The PCR primers used in this study were as follows: Forward, 5'-AGCTCGTGT CTCCCAGAGT-3' and reverse, 5'-CGTTCACGTTTGCAG AGATGT-3' for nephrin; forward, 5'-CTGGAGCTAAAG GACACACAGA-3' and reverse, 5'-GTGAAGGGACCCAAG CTCTC-3' for angiotensinogen (AGT) and forward, 5'-GGA TTTGGTCGTATTGGG-3' and reverse, 5'-GGAAGATGG TGATGGGATT-3' for GAPDH.

Western blotting. The kidney was homogenized and protein extracted using NP-40 buffer (Beyotime Institute of Biotechnology), followed by 5-10 min boiling and centrifugation $\left(4^{\circ} \mathrm{C}, 10 \mathrm{~min}, 12,000 \mathrm{x} \mathrm{g}\right)$ to obtain the 
A

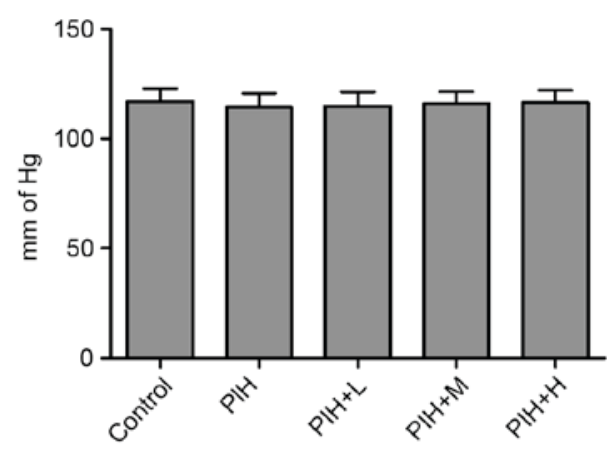

C

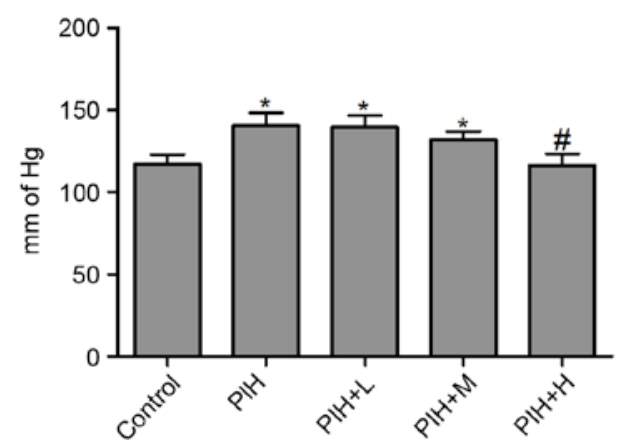

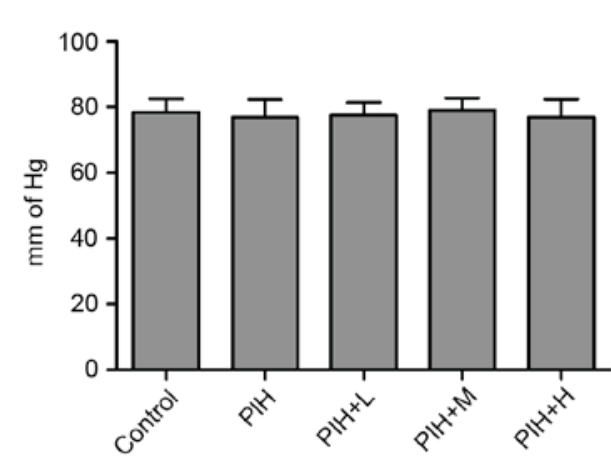

D

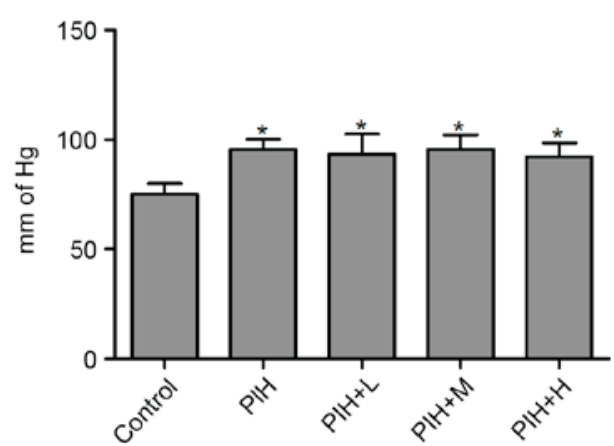

Figure 1. Effects of icariin on SBP and DBP in pregnant rats. (A) SBP and (B) DBP were measured on day 1 in pregnant rats. (C) SBP and (D) DBP were measured on day 18 in pregnant rats. Data are expressed as the mean \pm standard deviation. $n=8$ in each group. "P<0.05 vs. control; ${ }^{\prime \prime} \mathrm{P}<0.05$ vs. PIH group. SBP, systolic blood pressure; DBP, diastolic blood pressure; PIH, pregnancy-induced hypertension; L, low; M, medium; H, high.

supernatant. Protein concentrations were determined using the Bicinchoninic Acid kit for Protein Determination (Sigma-Aldrich; Merck KGaA). Samples containing $50 \mu \mathrm{g}$ protein were separated on $10 \%$ SDS-PAGE gel and transferred to nitrocellulose membranes (Bio-Rad Laboratories, Inc., Hercules, CA, USA). Following saturation with $5 \%$ (w/v) non-fat dry milk in TBS and $0.1 \%(w / v)$ Tween-20 (TBST), the membranes were incubated with the following primary antibodies, against nephrin (catalog no. sc-377246; 1:1,000; Santa Cruz Biotechnology, Inc.) and Ang II (catalog no. sc-20718; 1:500; Santa Cruz Biotechnology, Inc.) at $4^{\circ} \mathrm{C}$ overnight. Following 3 washes with TBST, membranes were incubated with secondary immunoglobulins (donkey anti-goat IgG; catalog no. sc-2020; 1:10,000; Santa Cruz Biotechnology, Inc.) conjugated to IRDye $800 \mathrm{CW}$ Infrared Dye (LI-COR Biosciences, Lincoln, NE, USA). Following a $2 \mathrm{~h}$ incubation period at $37^{\circ} \mathrm{C}$, membranes were washed 3 times with TBST. Blots were visualized using the Odyssey Infrared Imaging System (LI-COR Biosciences). Signals were densitometrically assessed (Odyssey Application Software, version 3.0; LI-COR Biosciences) and normalized to the GAPDH signals to correct for unequal loading (catalog no. sc-365062; 1:2,000; Santa Cruz Biotechnology, Inc.).

Statistical analysis. The data from these experiments are presented as the mean \pm standard deviation for each group. All statistical analyses were performed using PRISM version 4.0 (GraphPad Software, Inc., La Jolla, CA, USA). Inter-group differences were analyzed using one-way analysis of variance followed by Tukey's multiple comparison test as a post-hoc test to compare the group means. $\mathrm{P}<0.05$ was considered to indicate a statistically significant difference.

\section{Results}

Icariin regulates systolic blood pressure in PIH rats. Firstly, the SBP and DBP were measured in the control, PIH and icariin treated rats, and it was demonstrated that no significant differences were present in SBP (Fig. 1A) and DBP (Fig. 1B) among the five experimental groups on day 1. However, SBP and DBP were significantly increased in the PIH group compared with control group on day 18. The significant increase in SBP in $\mathrm{PIH}$ rats was prevented by icariin at a high concentration on day 18. However, icariin administration had no effect on DBP compared with PIH group on day 18 (Fig. 1C and D).

Icariin improves proteinuria in PIH rats. The urinary protein excretion increased $\sim$-fold in PIH rats compared with control pregnant rats (Fig. 2A). However, icariin administration reversed increased urinary protein in PIH rats at medium and high concentrations on day 18 (Fig. 2A). Furthermore, BUN and serum creatinine were significantly elevated in PIH rats compared with control pregnant rats. BUN and serum creatinine levels in PIH rats were attenuated following treatment with icariin at medium and high concentrations on day 18 (Fig. 2B and C). 
A

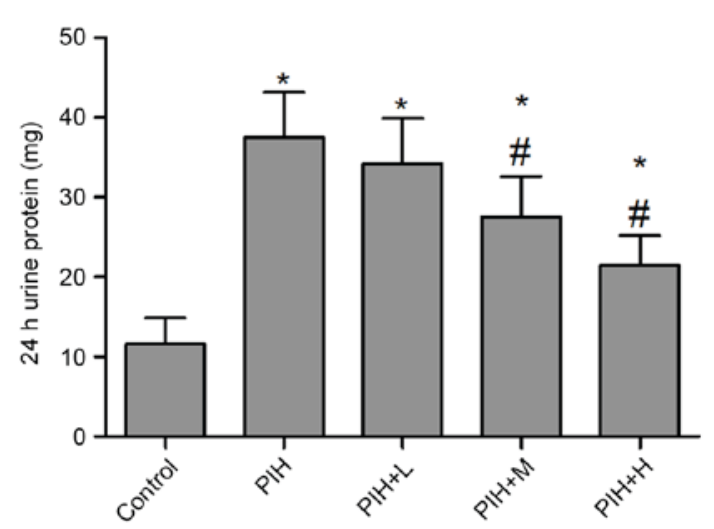

B

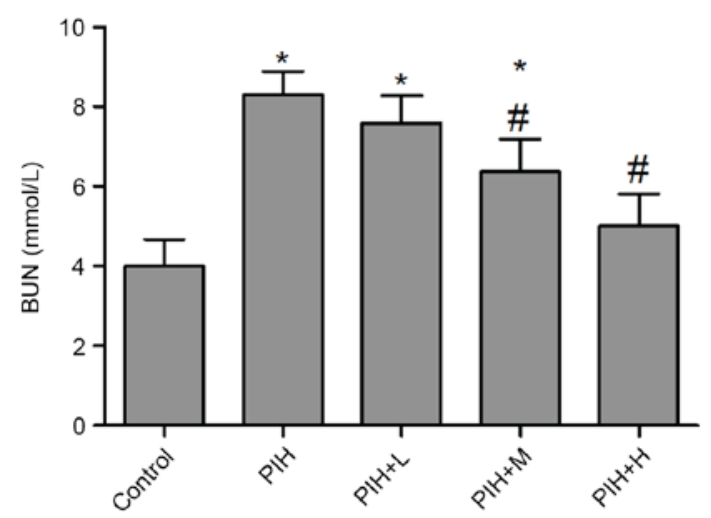

C

Day 18

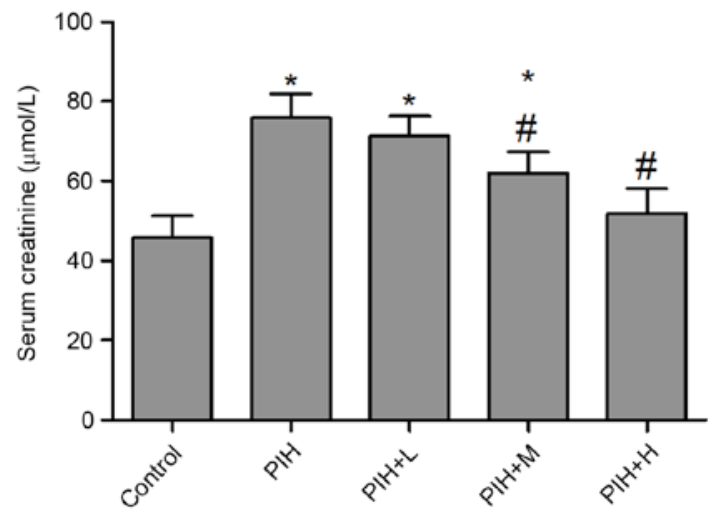

Figure 2. Effects of icariin on proteinuria in PIH rats. (A) $24 \mathrm{~h}$ urinary protein, (B) BUN and (C) serum creatinine were detected on day 18 in pregnant rats. Data are expressed as the mean \pm standard deviation. $n=8$ in each group. ${ }^{\mathrm{P}}<0.05$ vs. control; ${ }^{*} \mathrm{P}<0.05$ vs. PIH group. $\mathrm{BUN}$, blood urea nitrogen; $\mathrm{PIH}$, pregnancy-induced hypertension; L, low; M, medium; $\mathrm{H}$, high.

Icariin improves reproductive performance in PIH rats. Litters from PIH rats were significantly smaller in number compared with control pregnant rats, and icariin prevented the reduction in litter number induced by PIH (Fig. 3A). Furthermore, L-NAME administration (PIH group) resulted in a reduction in the average weight of the pup, however, icariin administration did not result in any significant weight gain of the pups during pregnancy (Fig. 3B).

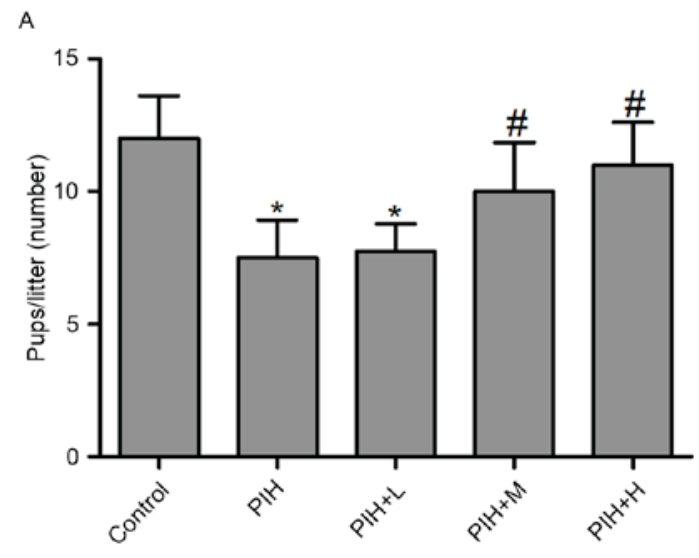

B

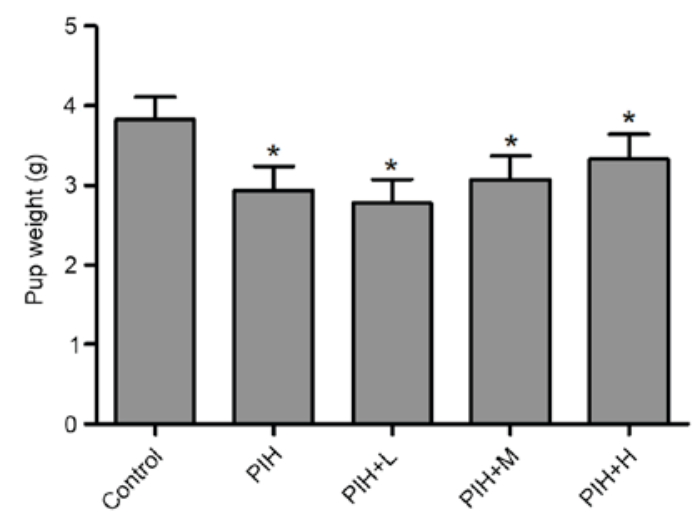

Figure 3. Effect of icariin on reproductive performance in PIH rats. Factors associated with reproductive performance including (A) litter number and (B) pup weight were measured across groups. Data are expressed as the mean \pm standard deviation. $\mathrm{n}=8$ in each group. ${ }^{*} \mathrm{P}<0.05$ vs. control; ${ }^{*} \mathrm{P}<0.05$ vs. PIH group. PIH, pregnancy-induced hypertension; L, low; M, medium; $\mathrm{H}$, high.

Icariin alleviates the pathological alterations of the kidney in PIH rats. H\&E staining demonstrated that L-NAME administration resulted in severe mesangial expansion and significant basement membrane thickening, however, kidney morphology was well preserved in control pregnant rats. Compared with the PIH group, rats with L-NAME-induced PIH treated with icariin (50 or $100 \mathrm{mg} / \mathrm{kg}$ ) exhibited markedly reduced severity of glomerular lesions (Fig. 4A). The podocyte protein nephrin is essential for maintaining the filtration barrier of the kidney and preventing proteinuria (16). A previous study indicated that glomerular expression of nephrin is decreased in kidney sections from women with pre-eclampsia (17). In the present study, the expression of nephrin was measured in the kidney from control pregnant and L-NAME administered PIH rats. The results demonstrated that L-NAME administration exerted a marked decrease in the mRNA and protein expression of nephrin in the kidneys, compared with the control group. However, icariin (50 or $100 \mathrm{mg} / \mathrm{kg}$ ) treatment significantly reversed L-NAME-induced downregulation of nephrin levels in the kidney (Fig. 4B and C).

Icariin inhibits Ang II activity in PIH rats. Previous studies suggest that the circulating and local renin-angiotensin systems 
A
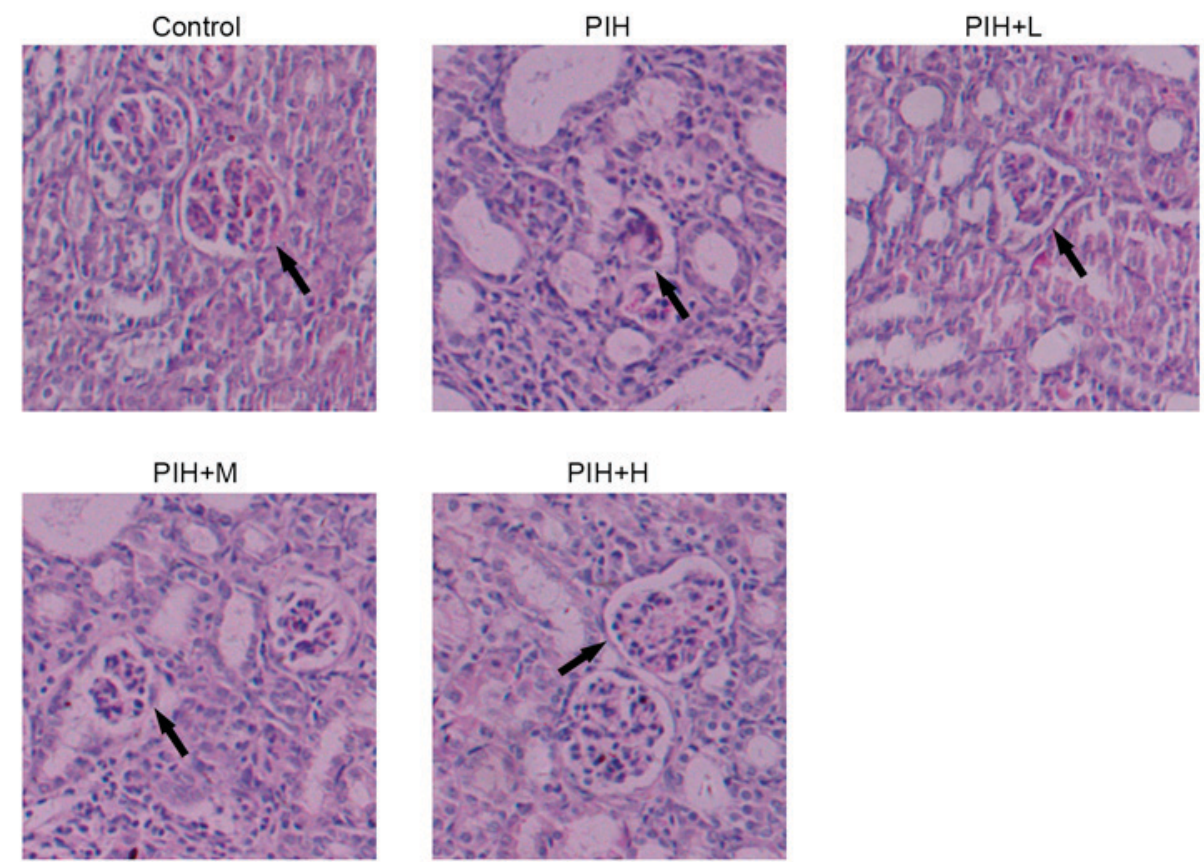

B

C
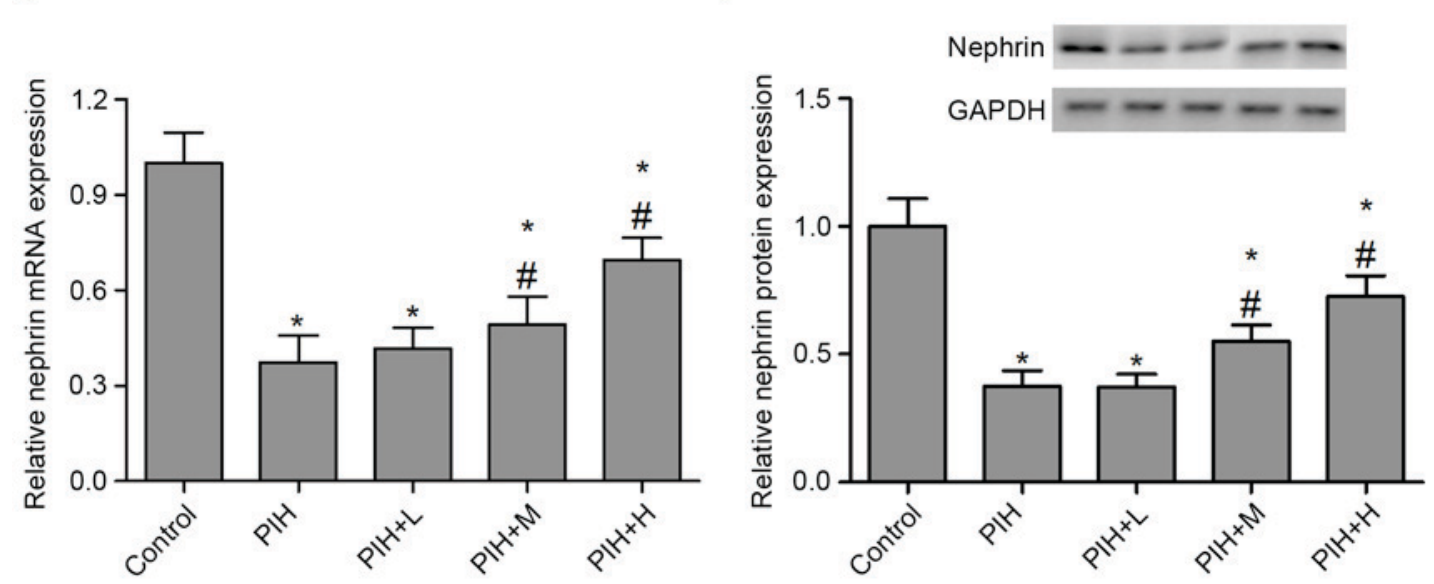

Figure 4. Effect of icariin on pathological alterations of the kidney in PIH rats. (A) Renal pathological hematoxylin \& eosin staining was performed following icariin treatment in PIH rats. Arrows indicate the location of glomerular legions. (B) Reverse transcription-quantitative polymerase chain reaction and (C) western blotting were performed to measure mRNA and protein levels of nephrin in the kidneys of PIH and normal pregnant rats. Data are expressed as the mean \pm standard deviation. $\mathrm{n}=8$ in each group. ${ }^{*} \mathrm{P}<0.05$ vs. control; ${ }^{*} \mathrm{P}<0.05$ vs. PIH group. Magnification, $\mathrm{x} 200$. PIH, pregnancy-induced hypertension; $\mathrm{L}$, low; M, medium; H, high.

(RAS) are activated during rat pregnancy $(18,19)$. However, the role of local RAS activity in the kidney of PIH rats remains to be elucidated. The present study investigated the renoprotective effects of icariin in PIH rats, and the association between icariin and the local renal RAS in the progression of PIH was examined. The expression of Ang II, as a key active peptide in the RAS, was measured in the kidney of control pregnant and PIH rats. The results demonstrated that circulating Ang II and AGT mRNA expression levels were significantly elevated in PIH rats compared with control pregnant rats. Rats that received icariin at the two greater doses (50 or $100 \mathrm{mg} / \mathrm{kg}$ ), exhibited alleviated circulating Ang II levels (Fig. 5A) and AGT mRNA expression (Fig. 5B), compared with the PIH group. Furthermore, the protein expression of Ang II was significantly increased in the kidneys of PIH rats compared with control group. However, icariin administration significantly reversed the L-NAME-induced upregulation of Ang II levels in the kidney (Fig. 5C). These effects of icariin on Ang II expression were verified by immunohistochemical staining (Fig. 5D).

\section{Discussion}

In the present study, the urinary protein excretion was significantly increased in PIH rats, and PIH-induced AKI resulted in profound renal histological alterations, including mesangial expansion and glomerular lesions, accompanied by increased BUN and serum creatinine. The protective effects of icariin in PIH-induced nephropathy were assessed. The results of the present study demonstrated that icariin administration suppressed urinary protein excretion and renal tissue damage 
A

Ang II in circulation
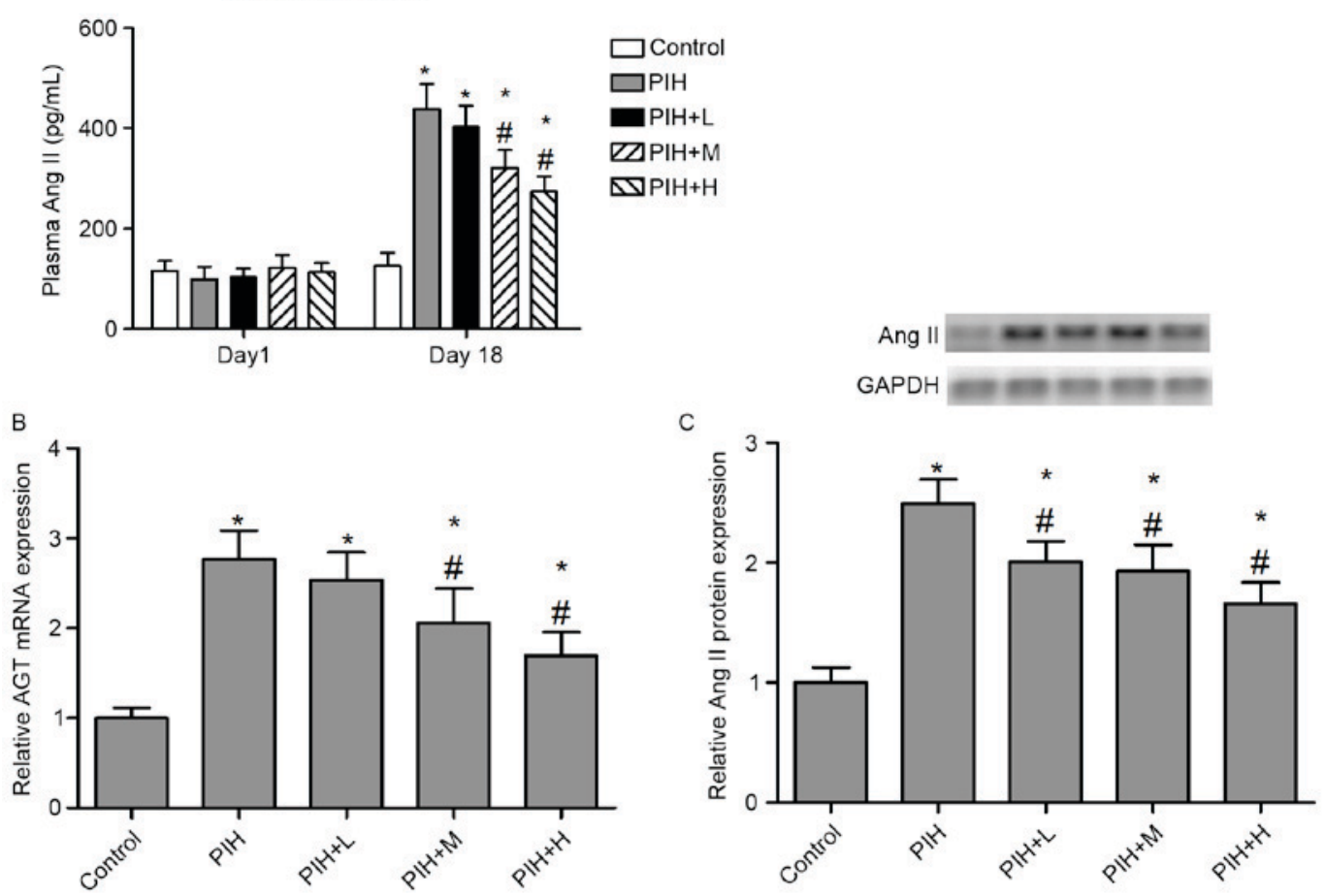

D
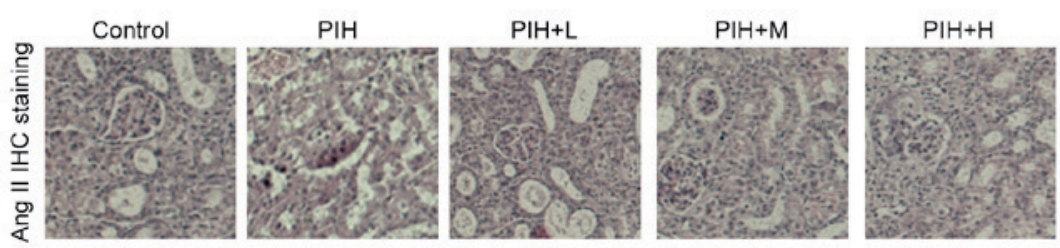

Figure 5. Effects of icariin on the renin angiotensin system. (A) Plasma levels of Ang II were detected using an ELISA assay. (B) AGT mRNA and (C) Ang II protein expression levels in the kidneys of PIH and normal pregnant rats were detected via reverse transcription-quantitative polymerase chain reaction and western blotting, respectively. (D) IHC staining analysis of kidney was evaluated using anti-rat Ang II. Magnification, x100. Data are expressed as the mean \pm standard deviation. $\mathrm{n}=8$ in each group. ${ }^{*} \mathrm{P}<0.05$ vs. control; ${ }^{*} \mathrm{P}<0.05$ vs. PIH group. Ang II, angiotensin II; AGT, angiotensinogen; IHC, immunohistochemical; PIH, pregnancy-induced hypertension; L, low; M, medium; H, high.

in PIH rats. Furthermore, upregulation of circulating and renal Ang II levels in PIH rats appeared to be reversed by icariin administration.

Pregnancy-induced hypertension has been accompanied by renal injury and proteinuria. Renal regeneration is at the center of treating AKI and other renal diseases (12). Previous studies suggest that icariin possesses the ability to promote the regeneration and differentiation of nephrocytes $(12,20)$. The present study hypothesized that icariin may improve PIH-induced renal injury and proteinuria. Histomorphological examinations demonstrated that icariin markedly improved glomerular lesions and renal interstitial fibrosis in PIH rats. Increased levels of BUN and serum creatinine in PIH rats were significantly reduced by icariin administration. Notably, icariin treatment significantly reversed the downregulation of nephrin mRNA and protein expression in the kidneys of PIH rats. Nephrin, a cytoskeletal protein which localizes to the slit pore of podocytes, may be important in proteinuria (21). An experimental model of diabetes and hypertension suggests that downregulation of nephrin levels in the kidney is associated with the development of albuminuria (22). The nephrin gene is specifically expressed in the kidney, brain and pancreas of rodents, and inactivation of nephrin results in proteinuria and neonatal death (23). Nephrin as a biomarker reflects podocyte dysfunction in various kidney disease models (24). The present study verified that L-NAME treatment in pregnant rats resulted in AKI, evidenced by severe proteinuria and BUN and serum creatinine elevation, which may potentially be associated with a decrease in nephrin expression in the kidney. Icariin suppressed the elevation of proteinuria, BUN and serum creatinine and increased the mRNA and protein expression levels of nephrin. The renoprotective effects of icariin may therefore be mediated via the upregulation of nephrin levels in the kidney.

RAS activation has previously been demonstrated to be important in the pathology of hypertension (25). However, its involvement in the pathogenesis of PIH remains to be fully elucidated. A previous study indicates that pregnant women with high susceptibility to increased Ang II levels had a high risk of developing pre-eclampsia (26). Notably, the circulatory RAS in pre-eclampsia appears to be suppressed (27), however the underlying mechanisms remain to be investigated. Various animal models demonstrate an increase in circulating maternal 
renin during gestation (28). A continuous infusion of high-dose Ang II during pregnancy in mice induces hypertension, proteinuria, intrauterine growth restriction and kidney injury (29). Consistent with the previous observations in rat pregnancy, the present study demonstrated that circulating and local renal Ang II expression were significantly elevated in PIH rats compared with control pregnant rats. These results suggest that increased Ang II activity may promote PIH in pregnant rats. Icariin administration significantly reversed the upregulation of Ang II levels in the plasma and kidney of PIH rats.

In conclusion, the findings of the present study suggest that icariin improves proteinuria and renal injury, and the underlying mechanism is mediated, in part, via the upregulation of nephrin expression and downregulation of Ang II. These results may therefore provide a novel therapeutic strategy for the treatment of PIH in the future.

\section{Acknowledgements}

The present study was supported by the Science and Technology Fund of Tianjin Municipal Health Bureau (grant no. 2013KZ024).

\section{References}

1. Veerbeek JH, Hermes W, Breimer AY, van Rijn BB, Koenen SV, Mol BW, Franx A, de Groot CJ and Koster MP: Cardiovascular disease risk factors after early-onset preeclampsia, late-onset preeclampsia and pregnancy-induced hypertension. Hypertension 65: 600-606, 2015.

2. Shortliffe LM, Hammam O, Han X, Kouba E, Tsao PS and Wang B: Dietary fructose in pregnancy induces hyperglycemia hypertension, and pathologic kidney and liver changes in a rodent model. Pregnancy Hypertens 5: 308-314, 2015.

3. Kendrick J, Sharma S, Holmen J, Palit S, Nuccio E and Chonchol M: Kidney disease and maternal and fetal outcomes in pregnancy. Am J Kidney Dis 66: 55-59, 2015.

4. Liu YM, Bao HD, Jiang ZZ, Huang YJ and Wang NS: Pregnancy-related acute kidney injury and a review of the literature in China. Intern Med 54: 1695-1703, 2015.

5. Carty DM, Delles C and Dominiczak AF: Preeclampsia and future maternal health. J Hypertens 28: 1349-1355, 2010.

6. Haase N, Golic M, Herse F, Rugor J, Linz D, Solano ME, Müller DN and Dechend R: Relaxin treatment in an Ang-II-based transgenic preeclamptic-rat model. PLoS One 11: e0150743, 2016.

7. Fan C, Yang Y, Liu Y, Jiang S, Di S, Hu W, Ma Z, Li T, Zhu Y and $\mathrm{Xin} Z$ : Icariin displays anticancer activity against human esophageal cancer cells via regulating endoplasmic reticulum stress-mediated apoptotic signaling. Sci Rep 6: 21145, 2016.

8. Wang J, Tao Y, Ping Z, Zhang W, Hu X, Wang Y, Wang L, Shi J, $\mathrm{Wu} \mathrm{X}$ and Yang $\mathrm{H}$ : Icariin attenuates titanium-particle inhibition of bone formation by activating the Wnt/ $\beta$-catenin signaling pathway in vivo and in vitro. Sci Rep 6: 23827, 2016.

9. Zhang G, Qin L and Shi Y: Epimedium-derived phytoestrogen flavonoids exert beneficial effect on preventing bone loss in late postmenopausal women: A 24-month randomized, double-blind and placebo-controlled trial. J Bone Miner Res 22: 1072-1079, 2007.

10. Xiao-Hong D, Chang-Qin X, Jian-Hua H, Wen-Jiang Z and Bing S: Icariin delays homocysteine-induced endothelial cellular senescence involving activation of the PI3K/AKT-eNOS signaling pathway. Pharm Biol 51: 433-440, 2013.

11. Ma P, Zhang S, Su X, Qiu G and Wu Z: Protective effects of icariin on cisplatin-induced acute renal injury in mice. Am J Transl Res 7: 2105-2114, 2015.

12. Huang Z, He L, Huang D, Lei S and Gao J: Icariin protects rats against 5/6 nephrectomy-induced chronic kidney failure by increasing the number of renal stem cells. BMC Complement Altern Med 15: 378, 2015.
13. Li YC, Ding XS, Li HM and Zhang C: Icariin attenuates high glucose-induced type IV collagen and fibronectin accumulation in glomerular mesangial cells by inhibiting transforming growth factor- $\beta$ production and signalling through $\mathrm{G}$ protein-coupled oestrogen receptor 1. Clin Exp Pharmacol Physiol 40: 635-643, 2013.

14. National Research Council (US) Committee for the Update of the Guide for the $\mathrm{C}$ and Use of Laboratory Animals: Guide for the Care and Use of Laboratory Animals, 8th edition. National Academies Press (US), Washington (DC), 2011.

15. Livak KJ and Schmittgen TD: Analysis of relative gene expression data using real-time quantitative PCR and the 2(-Delta Delta C(T)) method. Methods 25: 402-408, 2001.

16. Lin CL, Lee PH, Hsu YC, Lei CC, Ko JY, Chuang PC, Huang YT, Wang SY, Wu SL and Chen YS: MicroRNA-29a promotion of nephrin acetylation ameliorates hyperglycemia-induced podocyte dysfunction. J Am Soc Nephrol 25: 1698-1709, 2014.

17. Garovic VD, Wagner SJ, Petrovic LM, Gray CE, Hall P, Sugimoto H, Kalluri R and Grande JP: Glomerular expression of nephrin and synaptopodin, but not podocin, is decreased in kidney sections from women with preeclampsia. Nephrol Dial Transplant 22: 1136-1143, 2007.

18. Hering L, Herse F, Geusens N, Verlohren S, Wenzel K, Staff AC, Brosnihan KB, Huppertz B, Luft FC and Muller DN: Effects of circulating and local uteroplacental angiotensin II in rat pregnancy. Hypertension 56: 311-318, 2010.

19. Shah DM: Role of the renin-angiotensin system in the pathogenesis of preeclampsia. Am J Physiol Renal Physiol 288: F614-625, 2005.

20. Huang JH, Cai WJ, Zhang XM and Shen ZY: Icariin promotes self-renewal of neural stem cells: An involvement of extracellular regulated kinase signaling pathway. Chin J Integr Med 20: 107-115, 2014.

21. Takahashi A, Fukusumi Y, Yamazaki M, Kayaba M, Kitazawa Y, Tomita $\mathrm{M}$ and Kawachi $\mathrm{H}$ : Angiotensin II type 1 receptor blockade ameliorates proteinuria in puromycin aminonucleoside nephropathy by inhibiting the reduction of NEPH1 and nephrin. J Nephrol 27: 627-634, 2014.

22. Forbes JM, Bonnet F, Russo LM, Burns WC, Cao Z, Candido R, Kawachi H, Allen TJ, Cooper ME, Jerums G and Osicka TM: Modulation of nephrin in the diabetic kidney: Association with systemic hypertension and increasing albuminuria. J Hypertens 20: 985-992, 2002.

23. Putaala H, Soininen R, Kilpeläinen P, Wartiovaara $J$ and Tryggvason K: The murine nephrin gene is specifically expressed in kidney, brain and pancreas: Inactivation of the gene leads to massive proteinuria and neonatal death. Hum Mol Genet 10: 1-8, 2001.

24. Wada Y, Abe M, Moritani H, Mitori H, Kondo M, Tanaka-Amino K, Eguchi M, Imasato A, Inoki $Y$ and Kajiyama H: Original research: Potential of urinary nephrin as a biomarker reflecting podocyte dysfunction in various kidney disease models. Exp Biol Med (Maywood) 241: 1865-1876, 2016.

25. Solomon SD, Janardhanan R, Verma A, Bourgoun M, Daley WL, Purkayastha D, Lacourcière Y, Hippler SE, Fields H and Naqvi TZ: Effect of angiotensin receptor blockade and antihypertensive drugs on diastolic function in patients with hypertension and diastolic dysfunction: A randomised trial. Lancet 369: 2079-2087, 2007.

26. Seki H: The role of the renin-angiotensin system in the pathogenesis of preeclampsia - new insights into the renin-angiotensin system in preeclampsia. Med Hypotheses 82: 362-367, 2014.

27. Weir RJ, Paintin DB, Brown JJ, Fraser R, Lever AF, Robertson JI and Young J: A serial study in pregnancy of the plasma concentrations of renin, corticosteroids, electrolytes and proteins and of haematocrit and plasma volume. J Obstet Gynaecol Br Commonw 78: 590-602, 1971.

28. Irani RA and Xia Y: Renin angiotensin signaling in normal pregnancy and preeclampsia. Semin Nephrol 31: 47-58, 2011.

29. Shirasuna K, Karasawa T, Usui F, Kobayashi M, Komada T, Kimura H, Kawashima A, Ohkuchi A, Taniguchi S and Takahashi M: NLRP3 deficiency improves angiotensin II-induced hypertension but not fetal growth restriction during pregnancy. Endocrinology 156: 4281-4292, 2015. 